\title{
Quantum anomalous Hall effect and Anderson-Chern insulating regime in the noncollinear antiferromagnetic $3 Q$ state
}

\author{
Papa Birame Ndiaye, ${ }^{1,2, *}$ Adel Abbout, ${ }^{1}$ V. M. L. D. P. Goli, ${ }^{1}$ and Aurélien Manchon ${ }^{1,3, \dagger}$ \\ ${ }^{1}$ Physical Science and Engineering Division, King Abdullah University of Science and Technology, Thuwal 23955-6900, Saudi Arabia \\ ${ }^{2}$ Department of Physics, King Fahd University of Petroleum and Minerals, Dhahran 31261, Saudi Arabia \\ ${ }^{3}$ Computer, Electrical and Mathematical Science and Engineering Division, King Abdullah University of Science and Technology, \\ Thuwal 23955-6900, Saudi Arabia
}

(Received 22 July 2019; published 28 October 2019)

\begin{abstract}
We investigate the emergence of both quantum anomalous Hall and disorder-induced Anderson-Chern insulating phases in two-dimensional hexagonal lattices, with an antiferromagnetically ordered 3Q state and in the absence of spin-orbit coupling. Using tight-binding modeling, we show that such systems display not only a spin-polarized edge-localized current, the chirality of which is energy dependent, but also an impurityinduced transition from trivial metallic to topological insulating regimes, through one edge mode plateau. We compute the gaps' phase diagrams and demonstrate the robustness of the edge channel against deformation and disorder. Our study hints at the $3 \mathrm{Q}$ state as a promising building block for dissipationless spintronics based on antiferromagnets.
\end{abstract}

DOI: 10.1103/PhysRevB.100.144440

\section{INTRODUCTION}

Since control of the antiferromagnetic order parameter by electrical means was demonstrated [1,2], antiferromagnets have undoubtedly emerged as credible candidates for the replacement of ferromagnets as the active and upgrading spindependent element on which spintronic devices are based. With their numerous outstanding properties, they provide a rich playground to study unique magnetic properties combined with unconventional transport phenomena $[3,4]$. In particular, the interplay between electronic transport, topological properties of the ground states (in reciprocal space), and antiferromagnetic order in real space opens auspicious perspectives in the field of topological antiferromagnetic spintronics [5]. Indeed, although antiferromagnets break time-reversal symmetry locally, they are invariant under the combination of spin rotation and crystal symmetry operation (e.g., lattice translation in $G$-type collinear antiferromagnets, mirror symmetry in coplanar kagome antiferromagnets, etc.), which provides an analog to Kramers's degeneracy theorem. A direct consequence is that antiferromagnetism preserves the gapless states of topological materials such as topological insulators $[6,7]$ and Weyl semimetals $[8,9]$.

The search for topological effects has been particularly fruitful in the context of noncollinear antiferromagnets. For instance, the anomalous Hall effect in noncollinear but coplanar antiferromagnets was recently observed [10,11] and attributed to the coexistence of the spin Berry phase of the electronic ground states in the presence of spinorbit coupling (SOC) and symmetry breaking due to the noncollinear spin texture [12,13]. Along similar lines of

\footnotetext{
*papabirame.ndiaye@kfupm.edu.sa

aurelien.manchon@kaust.edu.sa
}

thought, large longitudinal and transverse spin currents in noncollinear coplanar antiferromagnets have also been reported [14]. Because they do not necessitate SOC, noncollinear, noncoplanar antiferromagnets constitute an appealing platform to sustain topological and trivial states and realize phase transitions between them. As a matter of fact, noncoplanar magnetic moments distributed on a lattice promote the emergence of the spin Berry phase even in the absence of SOC [15] and can experience topological phase transitions $[16,17]$.

Topological phase transitions in condensed matter have been intensively scrutinized over decades $[18,19]$. Such transitions are accompanied by the emergence of localized edge states unaffected by disorder and immune to backscattering, resulting in quantum (spin or anomalous) Hall effects in topological insulators [20]. The quantum anomalous Hall (QAH) effect is characterized by frictionless edge states in the absence of magnetic field. One way to fulfill this quantum state is to start with a time-reversal-symmetric $\left(\mathbb{Z}_{2}\right)$ topological insulator displaying the quantum spin Hall effect and break time-reversal symmetry by introducing magnetic order by magnetic doping $[21,22]$ or surface hybridization with a magnetic insulator $[23,24]$. This method applies to both strong and weak topological insulators (e.g., see Ref. [25]). Alternatively, Haldane showed that such a state can be obtained by engineering the band structure in such a way that conduction electrons experience a local, staggered magnetic flux that vanishes globally $[26,27]$. Such a situation can be achieved with the Kondo-lattice model on a triangular lattice $[28,29]$ or with the double-exchange model on a kagome lattice [30,31] where noncollinear, noncoplanar antiferromagnetism is stabilized and provides both nonvanishing Berry curvature and orbital gap. Nonetheless, only a limited number of works have focused on such materials [17,29,30], and a detailed understanding of quantum anomalous transport, the nature of 

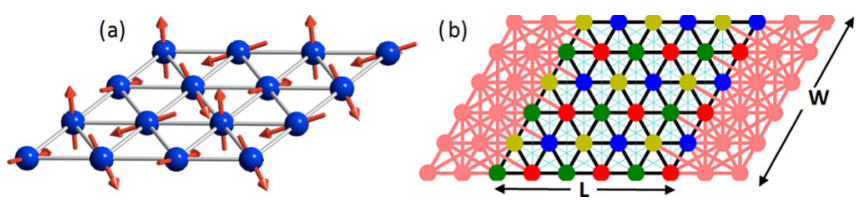

FIG. 1. (a) $3 \mathrm{Q}$ spin structure in a two-dimensional triangular lattice. (b) Leads plus system: the four magnetic atoms of the motif are in green, red, blue, and yellow. The thick black lines and the thin cyan lines are the first and second nearest-neighbor couplings, respectively. The pink regions refer to the leads. $L$ and $W$ indicate the length and the width of the system of study.

the edge states, and their robustness against disorder is still lacking.

In the present work, we exploit the noncollinear, noncoplanar antiferromagnetic texture in the absence of spin-orbit coupling to realize topologically protected edge transport. Taking the two-dimensional triangular lattice with $3 \mathrm{Q}$ spin texture as a prototype model, we show that the spin-polarized QAH effect is achieved without SOC in the zero-net-magnetization material, in agreement with previous predictions [17,29]. The chirality of the topological edge modes depends on the gap in which the Fermi energy is located. They are shown to be robust against disorder and unaffected by geometrical defects inside the lattice. We also investigate the rich next-nearestneighbor hopping phase diagram for the topological band gaps, opening around the high-symmetry Dirac points. Finally, we demonstrate that gradually tuned disorder can drive the 3Q antiferromagnetic metallic state into a topological Anderson-Chern insulating phase $[32,33]$, characterized by a conductance plateau of $e^{2} / h$.

\section{TRIANGULAR LATTICE MODEL}

Let us start with a two-dimensional hexagonal lattice underpinning the peculiar 3Q antiferromagnetic structure as depicted in Figs. 1(a) and 1(b): its three-dimensional chiral spin configuration, equivalent to a superposition of three spin spirals, is noncollinear, noncoplanar, and topologically nontrivial in real space while exhibiting no overall net magnetization. Such a magnetic state was predicted using $a b$ initio calculations by Sakuma [34] in the $\gamma$-FeMn disordered alloy and by Kurz et al. [35] at the $\mathrm{Mn} / \mathrm{Cu}(111)$ interface even in the absence of SOC. In the latter work, the authors attributed the onset of the $3 \mathrm{Q}$ state to the so-called four-spin interaction, i.e., an interaction involving all four magnetic moments of the magnetic unit cell [see Eq. (1)]. Further investigations in the context of the Kondo lattice and Hubbard model have indeed confirmed the thermodynamic stability of such a 3 Q spin texture in the triangular lattice $[28,29,36]$, pointing out the importance of itinerant electron contributions. To confirm the stability of the $3 \mathrm{Q}$ spin texture, we use atomistic spin modeling on a triangular lattice of $60 \times 60$ sites with an extended Heisenberg spin Hamiltonian of the form

$$
\begin{aligned}
\mathcal{H}= & J_{1} \sum_{\langle i, j\rangle} \mathbf{S}_{i} \cdot \mathbf{S}_{j}+J_{2} \sum_{\langle\langle i, j\rangle\rangle} \mathbf{S}_{i} \cdot \mathbf{S}_{j} \\
& +J_{4} \sum_{i j k l}\left[\left(\mathbf{S}_{i} \cdot \mathbf{S}_{j}\right)\left(\mathbf{S}_{k} \cdot \mathbf{S}_{l}\right)\right. \\
& \left.+\left(\mathbf{S}_{j} \cdot \mathbf{S}_{k}\right)\left(\mathbf{S}_{l} \cdot \mathbf{S}_{i}\right)-\left(\mathbf{S}_{i} \cdot \mathbf{S}_{k}\right)\left(\mathbf{S}_{j} \cdot \mathbf{S}_{l}\right)\right]
\end{aligned}
$$

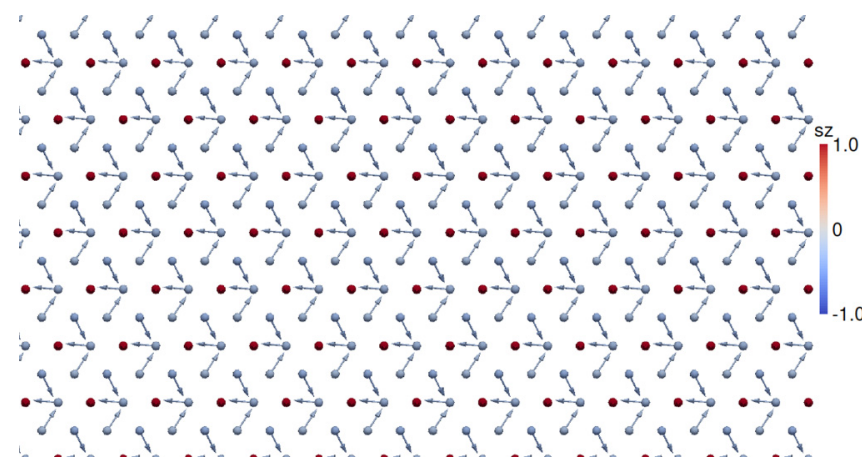

FIG. 2. The noncollinear 3Q magnetic structure of a triangular lattice with four-spin exchange interaction.

where $\mathbf{S}_{i}$ and $\mathbf{S}_{j}$ are normalized magnetic moment directions of sites $i$ and $j$, respectively. The first two terms in Eq. (1) are the nearest- and next-nearest-neighbor exchange energies with interactions $J_{1}$ and $J_{2}$, respectively. The last term is the four-spin exchange energy with $J_{4}$ as the four-spin exchange interaction [35]. All these interactions are antiferromagnetic. The magnetic properties of atomic spins are calculated using an atomistic spin simulation technique [37]. The spin dynamics is described by numerically solving the Landau-Lifshitz-Gilbert equation of the form

$$
\frac{\partial \mathbf{S}_{i}}{\partial t}=-\frac{\gamma}{1+\alpha^{2}}\left[\mathbf{S}_{i} \times \mathbf{H}_{i}^{\mathrm{eff}}+\alpha \mathbf{S}_{i} \times\left(\mathbf{S}_{i} \times \mathbf{H}_{i}^{\mathrm{eff}}\right)\right]
$$

where $\gamma=1.76 \times 10^{11} \mathrm{~T}^{-1} \mathrm{~s}^{-1}$ is the absolute value of the gyromagnetic ratio, $\alpha=0.1$ is the intrinsic Gilbert damping, and $\mathbf{H}_{i}^{\text {eff }}=-\left(1 / \mu_{s}\right) \partial_{\mathbf{s}_{i}} \mathcal{H}$ is the effective field experienced by the magnetic moment $\mathbf{S}_{i}$, with $\mu_{s}$ being the magnetic moment magnitude. To obtain the $3 \mathrm{Q}$ state, we consider a random spin configuration as an initial state, impose periodic boundary conditions, and scan through the parameter space $\left(J_{2} / J_{1}, J_{4} / J_{1}\right)$. The time evolution of Eq. (2) is solved using the Runge-Kutta fourth-order scheme, and the system is simulated for $10 \mathrm{~ns}$ to achieve energy convergence. The $3 \mathrm{Q}$ spin configuration is obtained for $J_{2}=0.3 J_{1}$ and $J_{4}=0.08 J_{1}$, as displayed in Fig. 2.

Now that the $3 \mathrm{Q}$ magnetic texture is confirmed as the ground state, let us consider electronic transport. The unit cell of the 3Q antiferromagnet has four chemically identical sites, tagged $\alpha, \beta, \gamma$, and $\delta$, as sketched in Fig. 3(a). The unit vectors of the magnetic moments on these sites fulfill $\mathbf{S}_{\alpha}+\mathbf{S}_{\beta}+\mathbf{S}_{\gamma}+\mathbf{S}_{\delta}=\mathbf{0}$. Remarkably, the associated spin chirality [16] reads $\kappa=\mathbf{S}_{\alpha} \cdot\left(\mathbf{S}_{\beta} \times \mathbf{S}_{\delta}\right)=-\mathbf{S}_{\alpha} \cdot\left(\mathbf{S}_{\beta} \times \mathbf{S}_{\gamma}\right) \neq 0$. In other words, the nonvanishing Berry flux threading the elementary trimer formed by neighboring sites [e.g., $\alpha, \beta, \delta$ sites in Fig. 3(a)] is compensated by the one threading the secondary trimer that involves one next-nearest neighbor (e.g., $\alpha, \beta, \gamma)$, so that the overall Berry flux vanishes over the magnetic cell, like in Haldane's model [26]. The associated effective magnetic flux [17] induces the anomalous Hall effect in frustrated ferromagnets [15], skyrmionic materials [38,39], and also 3Q antiferromagnets with no overall magnetism [29].

The two-dimensional hexagonal lattice, with the primitive cell formed by $\mathbf{a}_{1}=(1,0) a_{0}$ and $\mathbf{a}_{2}=(1 / 2, \sqrt{3} / 2) a_{0}$, and the first Brillouin zone with the reciprocal lattice spanned by 

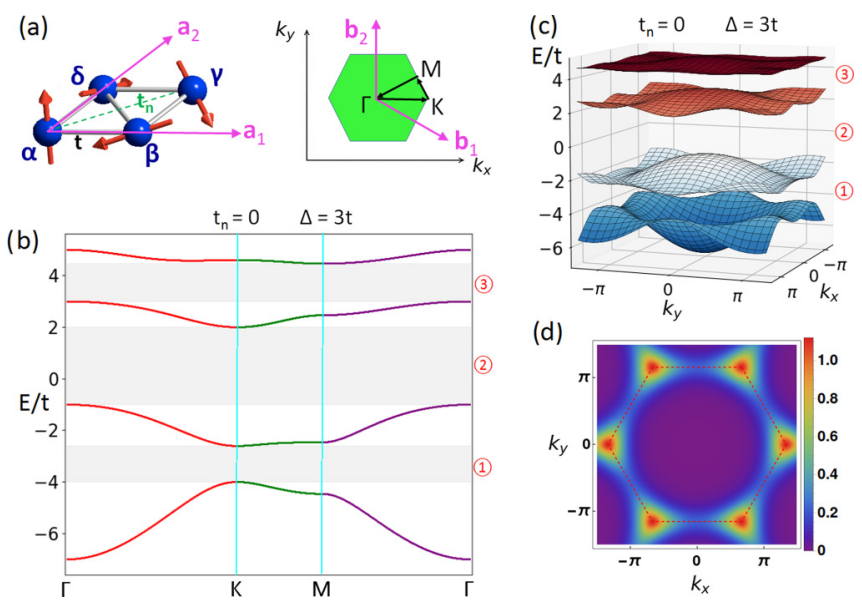

FIG. 3. (a) The unit cell with $\mathbf{S}_{\alpha}=(0,0,1), \quad \mathbf{S}_{\beta}=$ $(-2 \sqrt{2} / 3,0,-1 / 3), \quad \mathbf{S}_{\gamma}=(\sqrt{2} / 3,-\sqrt{6} / 3,-1 / 3), \quad$ and $\mathbf{S}_{\delta}=(\sqrt{2} / 3, \sqrt{6} / 3,-1 / 3)$, with the first Brillouin zone. (b) Band structure along the contour $\Gamma \rightarrow K \rightarrow M \rightarrow \Gamma$ for the model in Eq. (4) with $t_{n}=0$ and $\Delta=3 t$. (c) Bulk four-band structure plotted on $[-1.5 \pi, 1.5 \pi] \times[-1.5 \pi, 1.5 \pi]$. The circled numbers refer to the corresponding gap number: for example, the circled 1 refers to gap 1 in the text. (d) Trace of the Berry curvature tensor $\operatorname{Tr}\left[\overline{\bar{\Omega}}_{z}(\mathbf{k})\right]$, defined in Eq. (7), of the lowest degenerate band in (c) plotted within the same intervals.

$\mathbf{b}_{1}=(2 \pi,-2 \pi / \sqrt{3}) / a_{0}$ and $\mathbf{b}_{1}=(0,4 \pi / \sqrt{3}) / a_{0}$ are both depicted in Fig. 3(a). Here $a_{0}$ is the lattice constant taken as a unit of length in the following. The single-electron Hamiltonian under the tight-binding approximation reads

$$
\hat{H}=\sum_{i} \hat{c}_{i}^{\dagger}\left(\epsilon_{i}+\Delta \hat{\boldsymbol{\sigma}} \cdot \mathbf{S}_{i}\right) \hat{c}_{i}-t \sum_{\langle i, j\rangle} \hat{c}_{i}^{\dagger} \hat{c}_{j}-t_{n} \sum_{\langle\langle i, j\rangle\rangle} \hat{c}_{i}^{\dagger} \hat{c}_{j},
$$

where $\hat{c}_{i}^{\dagger}=\left(c_{i, \uparrow}^{\dagger}, c_{i, \downarrow}^{\dagger}\right)$, with $c_{i, \sigma}^{\dagger}$ being the creation operator for an electron with spin $\sigma$ on site $i, \hat{\sigma}$ is the vector of the Pauli matrices, $\Delta$ is the $s$ - $d$ exchange interaction between the itinerant spin and the local magnetic moment, and $t$ and $t_{n}$ are the nearest-neighbor and next-nearest-neighbor hopping parameters, respectively. Accordingly, $\sum_{i}$ runs over all the sites of the crystal, while $\sum_{\langle i, j\rangle}$ and $\sum_{\langle\langle i, j\rangle\rangle}$ run over the nearest-neighbor and the next-nearest-neighbor pairs, respectively. The eigenvalue problem can be recast in momentum space by Fourier transforming the real-space Hamiltonian $\hat{H}$ as $\hat{H}_{\mathbf{k}}\left|u_{n \mathbf{k}}\right\rangle=\varepsilon_{n \mathbf{k}}\left|u_{n \mathbf{k}}\right\rangle$, where $\hat{H}_{\mathbf{k}}$ is the $\mathbf{k}$-dependent Hamiltonian, $\varepsilon_{n \mathbf{k}}$ is the eigenenergy of the $n$th band, and, finally, $\left|u_{n \mathbf{k}}\right\rangle$ is the periodic part of the Bloch wave function. In the basis of the spinor $\psi_{\mathbf{k}}^{\dagger}=\left(\hat{c}_{\alpha \mathbf{k}}^{\dagger}, \hat{c}_{\beta \mathbf{k}}^{\dagger}, \hat{c}_{\delta \mathbf{k}}^{\dagger}, \hat{c}_{\gamma \mathbf{k}}^{\dagger}\right)$, the Hamiltonian in momentum space simply reads

$$
\hat{H}_{\mathbf{k}}=\left[\begin{array}{cccc}
\Delta \hat{\boldsymbol{\sigma}} \cdot \mathbf{S}_{\alpha} & L_{0} \hat{\mathbb{1}}_{2} & L_{1} \hat{\mathbb{1}}_{2} & L_{2} \hat{\mathbb{1}}_{2} \\
L_{0} \hat{\mathbb{1}}_{2} & \Delta \hat{\boldsymbol{\sigma}} \cdot \mathbf{S}_{\beta} & L_{2} \hat{\mathbb{1}}_{2} & L_{1} \hat{\mathbb{1}}_{2} \\
L_{1} \hat{\mathbb{1}}_{2} & L_{2} \hat{\mathbb{1}}_{2} & \Delta \hat{\boldsymbol{\sigma}} \cdot \mathbf{S}_{\gamma} & L_{0} \hat{\mathbb{1}}_{2} \\
L_{2} \hat{\mathbb{1}}_{2} & L_{1} \hat{\mathbb{1}}_{2} & L_{0} \hat{\mathbb{1}}_{2} & \Delta \hat{\boldsymbol{\sigma}} \cdot \mathbf{S}_{\delta}
\end{array}\right],
$$

with $L_{0}=-2 t \cos \left(\frac{1}{2} \mathbf{k} \cdot \mathbf{a}_{1}\right)-2 t_{n} \cos \left[\frac{1}{2} \mathbf{k} \cdot\left(\mathbf{a}_{1}-2 \mathbf{a}_{2}\right)\right], L_{1}=$ $-2 t \cos \left[\frac{1}{2} \mathbf{k} \cdot\left(\mathbf{a}_{1}-\mathbf{a}_{2}\right)\right]-2 t_{n} \cos \left[\frac{1}{2} \mathbf{k} \cdot\left(\mathbf{a}_{1}+\mathbf{a}_{2}\right)\right]$, and $L_{2}=$ $-2 t \cos \left(\frac{1}{2} \mathbf{k} \cdot \mathbf{a}_{2}\right)-2 t_{n} \cos \left[\frac{1}{2} \mathbf{k} \cdot\left(2 \mathbf{a}_{1}-\mathbf{a}_{2}\right)\right]$.

To conclude this section, let us indicate how the noncollinear magnetic moments generate an effective spin-orbit coupling. It is possible to rewrite Eq. (4) in the frame of the local quantization axes of the individual sites of the magnetic unit cell. In other words, by rewriting $\tilde{H}_{\mathbf{k}}=\hat{U}^{\dagger} \hat{H}_{\mathbf{k}} \hat{U}$, with

$$
\hat{U}=\left[\begin{array}{cccc}
\Delta \hat{\boldsymbol{\sigma}} \cdot \mathbf{n}_{\alpha} & 0 & 0 & 0 \\
0 & \Delta \hat{\boldsymbol{\sigma}} \cdot \mathbf{n}_{\beta} & 0 & 0 \\
0 & 0 & \Delta \hat{\boldsymbol{\sigma}} \cdot \mathbf{n}_{\gamma} & 0 \\
0 & 0 & 0 & \Delta \hat{\boldsymbol{\sigma}} \cdot \mathbf{n}_{\delta}
\end{array}\right],
$$

where $\mathbf{n}_{i}=\left(\mathbf{z}+\mathbf{S}_{i}\right) /\left|\mathbf{z}+\mathbf{S}_{i}\right|$, we obtain

$$
\tilde{H}_{\mathbf{k}}=\left[\begin{array}{cccc}
\Delta \hat{\sigma}_{z} & \tilde{t}_{\alpha \beta} & \tilde{t}_{\alpha \gamma} & \tilde{t}_{\alpha \delta} \\
\tilde{t}_{\beta \alpha} & \Delta \hat{\sigma}_{z} & \tilde{t}_{\beta \gamma} & \tilde{t}_{\beta \delta} \\
\tilde{t}_{\gamma \alpha} & \tilde{t}_{\gamma \beta} & \Delta \hat{\sigma}_{z} & \tilde{t}_{\gamma \delta} \\
\tilde{t}_{\delta \alpha} & \tilde{t}_{\delta \beta} & \tilde{t}_{\delta \gamma} & \Delta \hat{\sigma}_{z}
\end{array}\right] .
$$

In this representation, the diagonal part of the Hamiltonian is spin diagonal (no spin mixing occurs on the magnetic sites), and the information of the spin texture has been transferred to the hopping matrix elements $\tilde{t}_{i j}$. Explicitly, $\tilde{t}_{i j}=t_{i j}\left[\mathbf{n}_{i} \cdot \mathbf{n}_{j}+\right.$ $i \hat{\boldsymbol{\sigma}} \cdot\left(\mathbf{n}_{i} \times \mathbf{n}_{j}\right)$ ], where $t_{i j}$ is the off-diagonal matrix element of Eq. (4) at position $(i, j)$. In other words, the hopping matrix elements become complex and spin dependent, mimicking the action of spin-orbit coupling and promoting anomalous transport.

\section{SPIN BERRY PHASE INDUCED ANOMALOUS TRANSPORT}

\section{A. Topological edge states}

The band structure along the high-symmetry point contour and its three-dimensional version over the whole Brillouin zone are displayed in Figs. 3(b) and 3(c), respectively. There are four doubly degenerate bands, as dictated by Kramers's degeneracy theorem. Indeed, the four magnetic moments are oriented towards the four corners of a tetrahedron, and performing a rotation $\mathcal{R}$ within this tetrahedron is equivalent to performing a translation $T_{a}$ in the crystal lattice. Therefore, the operation $\mathcal{O}=\mathcal{R} T_{a}$ is a symmetry of the magnetic system. Upon time-reversal operation $\mathcal{T}$, the combined operator $\mathcal{T} \mathcal{O}$ is antiunitary, $(\mathcal{T O})^{2}=-1$, implying double degeneracy of the bands. Besides the antiferromagnetic gap appearing in the middle (denoted gap 2), two additional gaps are obtained in Figs. 3(b) and 3(c), referred to as gap 1 for the lower one and gap 3 for the upper one.

As mentioned in the Introduction, the origin of the QAH effect is rooted in the existence of a nonvanishing $k$-space Berry curvature defined as $\boldsymbol{\Omega}(\mathbf{k})=i\left\langle\nabla_{\mathbf{k}} u(\mathbf{k})|\times| \nabla_{\mathbf{k}} u(\mathbf{k})\right\rangle$ for a nondegenerate state. In the case of band degeneracy, the Berry curvature is not a vector anymore but adopts a tensorial form [40,41]. As a matter of fact, the time evolution of the system entails the occurrence of adiabatic transitions between states of the same subspace, and therefore, the Berry curvature tensor is constructed by all the wave function projections between the elements of the same subspace,

$$
\begin{aligned}
\overline{\overline{\mathbf{\Omega}}}_{i j}(\mathbf{k})= & i\left\langle\nabla_{\mathbf{k}} u_{i}(\mathbf{k})|\times| \nabla_{\mathbf{k}} u_{j}(\mathbf{k})\right\rangle \\
& +i \sum_{l \in \zeta}\left\langle u_{i}(\mathbf{k}) \mid \nabla_{\mathbf{k}} u_{l}(\mathbf{k})\right\rangle \times\left\langle u_{l}(\mathbf{k}) \mid \nabla_{\mathbf{k}} u_{j}(\mathbf{k})\right\rangle
\end{aligned}
$$


where $\zeta$ is the degenerate subspace. The second term encodes the signature of the non-Abelian topology of the covariant tensor. In this noncollinear, noncoplanar antiferromagnet, the bands are twofold degenerate and hence, the Berry curvature tensor for each degenerate band is a $2 \times 2$ nonvanishing matrix. As a matter of fact, close to gaps 1 and 3 , a nonvanishing $k$-space Berry curvature tensor emerges around the $K$ points [see Fig. 3(d)], enabling the onset of gapless chiral states at the edges. The physical origin of the Berry curvature is the exchange interaction that makes the spin of the conduction electrons align with the local moments, inducing a Berry curvature which gives rise to an anomalous velocity $[15,17]$. The Berry curvature tensor is not gauge invariant, and this leads to the appearance of jumps in the eigenvalues profile of the Berry curvature tensor when the gauge is not suitably chosen [41]. However, these jumps do not prevent from defining observables which are gauge invariant such as the determinant $\operatorname{det}[\overline{\bar{\Omega}}(\mathbf{k})]$ and the trace $\operatorname{Tr}[\overline{\bar{\Omega}}(\mathbf{k})]$. In the insulating regime, the anomalous Hall conductivity $\sigma_{x y}$ equals the integral of $\operatorname{Tr}[\overline{\bar{\Omega}}(\mathbf{k})]$ on the first Brillouin zone,

$$
\begin{gathered}
\sigma_{x y}=-\left(e^{2} / \hbar\right) \sum_{n} \int_{\mathrm{BZ}} d^{2} \mathbf{k} /(2 \pi)^{2} \operatorname{Tr}\left[\overline{\bar{\Omega}}_{n}(\mathbf{k})\right] f_{n}(\mathbf{k}) \\
=-\left(e^{2} / h\right) \mathcal{C}
\end{gathered}
$$

where $f_{n}(\mathbf{k})$ is the Fermi distribution for the degenerate states, $n$ is the index of the degenerate subspace, and $\mathcal{C}$ is the Chern number. The nonvanishing quantized $\sigma_{x y}$ in gaps 1 and 3 is the signature of the nontrivial topology of the $3 \mathrm{Q}$ state which is exposed by the presence of edge states. This property is absent in collinear as well as coplanar antiferromagnets.

For further insight into the character of these edge currents, we use a suitable tight-binding code for quantum transport [42] to build a translationally invariant nanowire along the $x$ direction while fixing the width $W$ to $50 a_{0}$ in order to ensure a large number of transport modes $N$. The calculations are performed at zero temperature in the ballistic regime. The one-dimensional band structure is plotted in Fig. 4(a), with the parameters of the system being $t_{n}=0.2 t$ and $\Delta=3 t$. This band structure exhibits four metallic regions characterized by a large number of bands and separated by two topological gaps

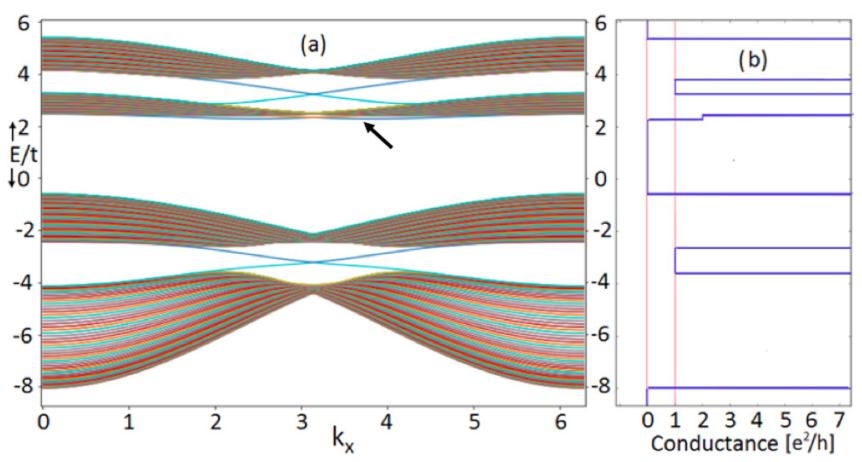

FIG. 4. (a) The one-dimensional band structure with the emergence of the gapless edge states seen in gaps 1 and 3 , with $t_{n}=0.2 t$, $\Delta=3 t$. The black arrow indicates the trivial edge states discussed in the main text. (b) Close-up of the corresponding conductance (in units of $\left.e^{2} / h\right)$.
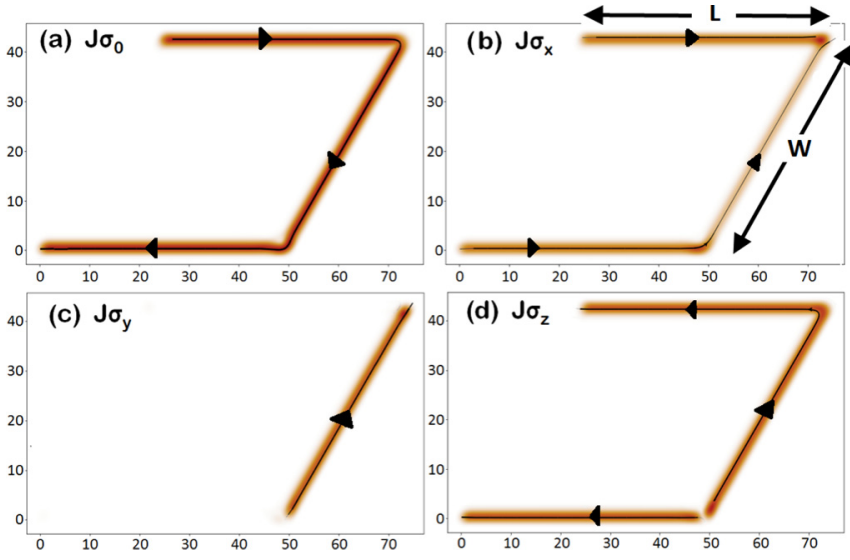

FIG. 5. (a) The unidirectional chiral edge current in the system (for an energy lying in gap 1 with a length and width of $L=W=$ $50 a_{0}$ and $t_{n}=0.2 t, \Delta=3 t$ ). The black arrows show the direction of the current. (b), (c), and (d) The spin polarization along $x, y$, and $z$, respectively: the charge current follows the net orientation of the edge spins.

(gap 1 and gap 3) where the conducting edge channels clearly appear and one trivial gap in the middle of the band structure (gap 2). Figure 4(b) displays the corresponding conductance $G=N \times e^{2} / h$ across the band structure. In the metallic regions, the large conductance reflects the large number of transport modes of the system $(N \gg 1)$. More interestingly, the conductance vanishes in the trivial gap (gap 2), while it saturates to $e^{2} / h$ in the topological gaps (gaps 1 and 3), reflecting the presence of a single chiral edge mode $(N=1)$. We notice that the conductance reaches $2 e^{2} / h$ around the transport energy $E=2.5 t$. This peculiarity is associated with the emergence of trivial, nonchiral edge states at the verge of the metallic regime, as indicated by the black arrow in Fig. 4(a).

To better understand the nature of the edge mode giving rise to the quantum conductance, we disconnect the right lead and compute the spatial profile of the local charge and spin currents for electrons coming from the left lead and bouncing back to it. The access to the local charge and spin currents flowing between any two sites $i$ and $j$ of a discretized lattice is a well-established procedure involving the hopping matrix between the two sites. From the real-space wave function $\psi_{i, j}$ as well as the hopping Hamiltonian $\hat{H}_{i j}$ between sites $i$ and $j$, the local spin current is naturally defined as $J_{\hat{\sigma}}^{i j}=$ $i\left(\psi_{j}^{\dagger} \hat{H}_{i j}^{\dagger} \hat{\boldsymbol{\sigma}} \psi_{i}-\psi_{i}^{\dagger} \hat{\boldsymbol{\sigma}} \hat{H}_{i j} \psi_{j}\right)$. The various components of the spin current are denoted $J \sigma_{\mathrm{x}}, J \sigma_{\mathrm{y}}$, and $J \sigma_{\mathrm{z}}$, and the charge current $J \sigma_{0}$ is obtained by replacing $\hat{\sigma}$ by $\hat{\mathbb{1}}_{2}$.

Figure 5(a) shows the local charge current $J \sigma_{0}$ when the operating energy is in the lower gap, gap 1: the unidirectional chiral edge state is propagating clockwise from the top edge to the bottom one, demonstrating the realization of the QAH effect in the absence of the spin-orbit coupling. On the other hand, when the Fermi energy of injected electrons is within the upper gap, gap 3, the same QAH effect is observed. In gap 3, however, the single propagating edge mode has a chirality opposite to the one from its gap 1 counterpart (counterclockwise from the bottom edge to the top one). 
The associated spin currents $J \sigma_{\mathrm{x}, \mathrm{y}, \mathrm{z}}$ are plotted in Figs. 5(b)-5(d), which clearly show that the charge current displayed in Fig. 5(a) is spin polarized along the net magnetization of the edge line. On the top edge, we chose the magnetic moments at the boundary to be $\mathbf{S}_{\gamma}$ and $\mathbf{S}_{\delta}$ [Fig. 3(a)]. Therefore, the net magnetization at this edge is directed along $\mathbf{S}_{\gamma}+\mathbf{S}_{\delta}=(\sqrt{2} / 3,0,-2 / 3)$. As a result, the spin current polarized along $x$ and $z$, while $J \sigma_{\mathrm{y}}=0$. Furthermore, $J \sigma_{\mathrm{x}}$ has the same direction as $J \sigma_{0}$, whereas $J \sigma_{\mathrm{z}}$ is opposite. On the right edge, we chose the magnetic moments at the boundary to be $\mathbf{S}_{\beta}$ and $\mathbf{S}_{\gamma}$ [Fig. 3(a)], which provides a net magnetization on the right edge directed along $\mathbf{S}_{\beta}+\mathbf{S}_{\gamma}=$ $(-\sqrt{2} / 3,-\sqrt{6} / 3,-2 / 3)$. The three negative components are seen in the spin polarization of the charge current on the right edge. Finally, the bottom edge has a net magnetization of $\mathbf{S}_{\alpha}+\mathbf{S}_{\beta}=(-2 \sqrt{2} / 3,0,2 / 3)$, as directly reflected in the spin polarization displayed in Figs. 5(b)-5(d).

\section{B. Engineering the band structure}

While each nearest-neighbor triangular plaquette bears a spin Berry phase $\kappa=\mathbf{S}_{\alpha} \cdot\left(\mathbf{S}_{\beta} \times \mathbf{S}_{\delta}\right)$, turning on the nextnearest-neighbor hopping $t_{n}$ creates new triangular plaquettes with opposite flux $\mathbf{S}_{\alpha} \cdot\left(\mathbf{S}_{\beta} \times \mathbf{S}_{\gamma}\right)=-\kappa[\alpha, \beta, \delta$, and $\gamma$ follow a counterclockwise circular ordering, as seen previously in Fig. 3(a)]. Therefore, by tuning the relative strength between the nearest-neighbor hopping $t$, the second-nearest-neighbor hopping $t_{n}$, and the exchange coupling $\Delta$, one can modify the emergent magnetic flux threading the magnetic unit cell and thereby engineer the band structure and its properties. The conditions on $\Delta$ and $t_{n}$ (both in units of $t$ ) to obtain a topologically nontrivial system are summarized in Figs. 6(a) and 6(b): the regions where a topological gap (gaps 1 and 3) exists are on a colored scale; the darker the color is, the larger the gap is. The white color in the phase diagrams denotes the absence of gap for the set of parameters concerned. The gaps plotted in the phase diagram are global ones; they represent the difference between the lowest energy of the $n$th band and the highest energy of the $(n-1)$ th band as computed over the whole Brillouin zone.

The size of gap 1 at $K=( \pm 4 \pi / 3,0)$ is displayed in Fig. 6(c) for different exchange couplings $\Delta$ as a function of $t_{n}$ : remarkably, gap 1 closes at $t_{n}=0.5 t$, which corresponds to the appearance of a Dirac cone at $K$. This can also be viewed in Fig. 6(a), at the dashed line. When $t_{n}=0.5 t$, global gap 3 vanishes [see dashed line in Fig. 6(b)]. However, at this condition there is another Dirac cone at $K$ and a local and direct band crossing around the same energy range as gap 3. As a matter of fact, by expanding the energy dispersion close to the $K$ point and around $t_{n} \approx 0.5 t$, we obtain for these two different Dirac cones $E_{K}^{ \pm}(q)= \pm \Delta \pm \frac{\sqrt{3}}{4} t|q| a_{0}$, and $|q|$ is the norm of the momentum $q$ around the $K$ point. Notice that Fig. 6(c) shows a finite local gap when $\Delta=0.5 t$ and for $-t<t_{n}<0.5 t$, whereas Fig. 6(a) denies the existence of a global gap for these conditions. The bands are, in fact, overlapping and cross indirectly at different $k$ points of the Brillouin zone, therefore ensuring the existence of a local gap despite the absence of global gaps.

Another remarkable feature appears in gap 1 when $t_{n} \rightarrow t$. This time, the Dirac cones emerge at $M=(\pi, \pi / \sqrt{3})$, as (a) Gap 1

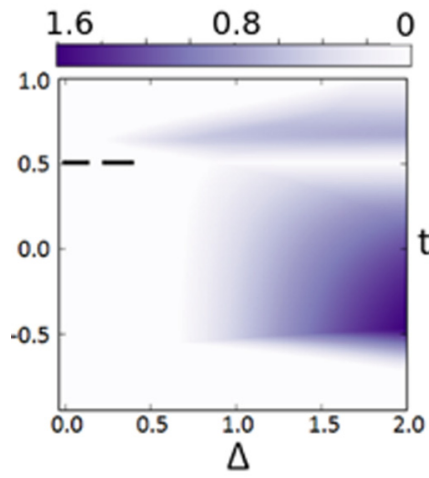

(c) Gap 1 at K point

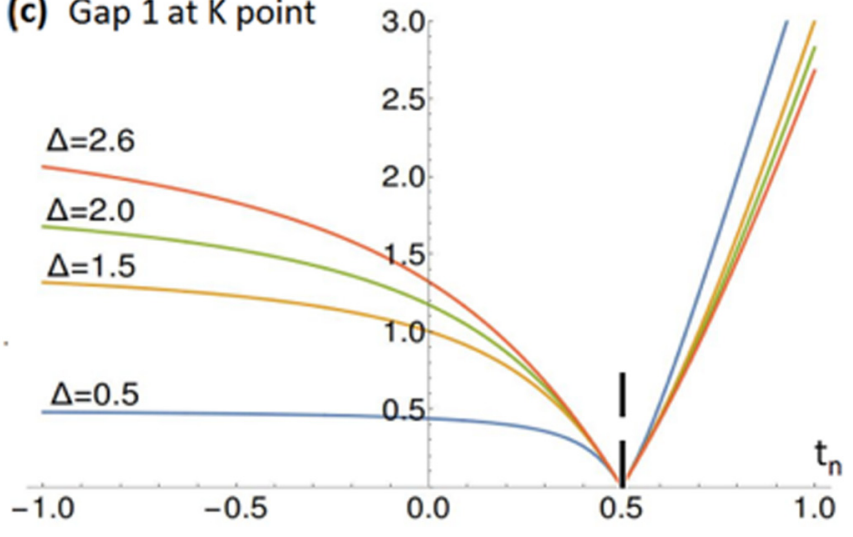

FIG. 6. (a) and (b) Phase diagrams for global topological gaps 1 and 3 (in units of $t$ ). $\Delta$ and $t_{n}$ are in units of $t$. (c) Local gap 1 at $K=(4 \pi / 3,0)$ as a function of $t_{n}$ and for four $\Delta$. The Dirac point appears at $K$ for $t_{n}=0.5 t$.

seen in the band structure in Fig. 7(a), located at the energy $E_{M}^{-}$. The Berry curvature calculation of the lowest-energy band, depicted in Fig. 7(b), confirms $M$ is the Dirac point around which the finite curvature is concentrated. Global gap 3 remains nonexistent for $t_{n} \rightarrow t$, as predicted in the phase diagram [Fig. 6(b)], but there is a Dirac-like crossing point at the energy $E_{M}^{+}$, as indicated in Fig. 7(a).

The position of the Dirac cones in reciprocal space is dictated by the symmetries of the Bloch states. For instance, in graphene the Dirac cone must be pinned on the $K$ and $K^{\prime}$ points due to time-reversal symmetry. In our case, the system
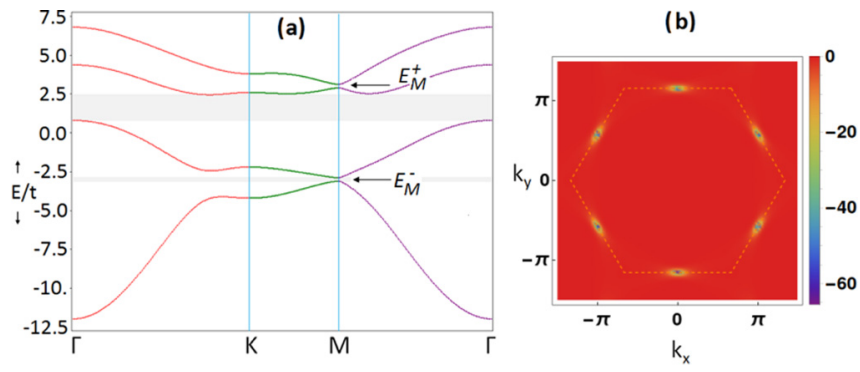

FIG. 7. (a) Band structure with $t_{n}=0.9 t$ and $\Delta=3 t$, with the Dirac point about to appear at $M$ when $t_{n} \rightarrow t$. (b) Trace of the Berry curvature tensor $\operatorname{Tr}[\overline{\bar{\Omega}}(\mathbf{k})]$ of the lowest band for the same set of parameters. 
TABLE I. Conditions under which fourfold degeneracy occurs in the Brillouin zone.

\begin{tabular}{cc}
\hline \hline Condition & Coordinates \\
\hline$t_{n}=-t$ & $\Gamma:(0,0)$ \\
$t_{n}=t / 2$ & $K, K^{\prime}:( \pm 4 \pi / 3,0)$ \\
$t_{n}=+t$ & $M, M^{\prime}:( \pm \pi, \pi / \sqrt{3})$ \\
\hline \hline
\end{tabular}

exhibits an analog of Kramers degeneracy but no time-reversal symmetry. Therefore, if a Dirac cone were to emerge, it would not need to be pinned on the $K$ point. In fact, Hou and Chen [43] recently showed that the position of a Dirac cone is determined by a so-called hidden symmetry. In a nutshell, Dirac cones appear at the $k$ points that are invariant under this hidden symmetry and whose eigenstates are doubly degenerate. The difficulty lies in identifying this hidden symmetry, which is not a trivial task. Alternatively, we provide a heuristic argument based on the structure of the Hamiltonian, Eq. (4). One (nonunique) way to obtain fourfold degeneracy is to cancel the off-diagonal hopping terms, i.e., $L_{1}=L_{2}=L_{0}=0$. Under such a condition, $\hat{H}_{\mathbf{k}}$ becomes block diagonal with two fourfold-degenerate eigenvalues at $\pm \Delta$. It is worth noticing that this degeneracy is due to the electronic hopping properties and not to the form of the magnetic texture. The position of the degeneracies in reciprocal space can be obtained directly from the above condition. We find that such degeneracies appear only at five possible $k$ points and for very specific values of the next-nearest-neighbor hopping parameter $t_{n}$. All these points are high-symmetry points of the hexagonal Brillouin zone and are summarized in Table I. Following this heuristic argument, the positions of the fourfold degeneracies are in agreement with the positions of the Dirac cones obtained numerically in Figs. 6(c) and 7(a).

\section{Robustness and Anderson-Chern insulator}

We now turn our attention towards the impact of Anderson disorder on the topological transport. For this purpose, we add to Eq. (3) nonmagnetic quenched (immobile) impurities, which are described as random on-site energies all over the sample, with a uniform distribution in the range $\left[-V_{\text {imp }}, V_{\text {imp }}\right]$ [39]. The calculated conductance of the strip is averaged over 1280 disorder configurations. The conductance $G$ as a function of disorder strength $V_{\text {imp }}$ is plotted in Fig. 8(a) for three different widths $W$ and setting the value of Fermi energy in topological gap $1\left(t_{n}=0.2 t, \Delta=3 t\right)$. The quantized conductance is robust for impurity strength ranging from 0 to $\sim 2 t$, showing that the topological edge currents are preserved even under relatively strong disorder: the regime remains ballistic. Above $V_{\mathrm{imp}} \approx 2 t$, the conducting edge channel is progressively destroyed, and the conductance starts decreasing significantly as the reflection probability becomes larger. As seen in Fig. 8(a), beyond the threshold disorder strength, the probability of backscattering naturally reduces as the width of the system becomes larger. The decaying part is proportional to the density of impurities for sufficiently large widths (not shown). Figure 8(b) furthermore demonstrates that these QAH states persist under geometrical deformations of the system's edges. In our case, the geometrical defects

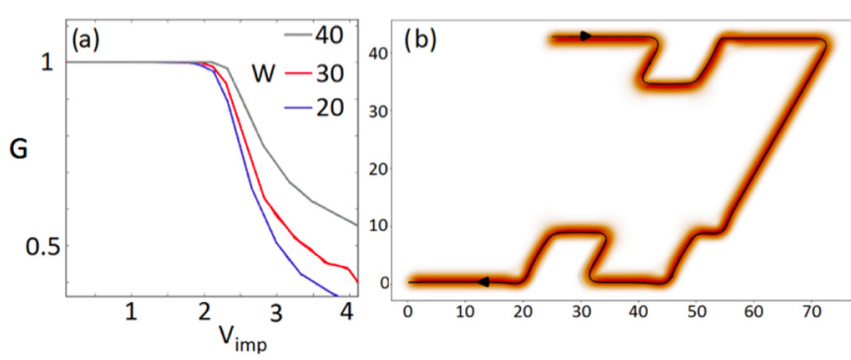

FIG. 8. (a) The conductance $G$ in units of $e^{2} / h$ as a function of the disorder strength when the Fermi energy is in gap $1(N=1)$ for three different widths $W=20 a_{0}, 30 a_{0}, 40 a_{0}$ and for $L=50 a_{0}$. (b) Robustness of the unidirectional chiral edge current against the local geometrical defects.

are multiple-site vacancy defects of different shapes at the boundaries of the periodic sample. In such a case, the system is not uniform anymore and displays geometrical singularities such as holes and geometrical cusps. Despite their presence, the unidirectional transport enabled by the band topology is immune to the backscattering due to local defects and singularities.

Finally, we show that in this metallic $3 \mathrm{Q}$ antiferromagnet, disorder is capable of inducing another nontrivial quantum phase, termed the topological Anderson-Chern insulator. Anderson's theory of localization states that strong disorder can promote metal-insulator transition [44]. In systems possessing nonvanishing Berry curvature, the renormalization of the topological effective mass and chemical potential induced by Anderson disorder favors the emergence of topological edge states when transiting towards the insulating regime [32,33]. A similar effect is expected in the present case, where the nontrivial band structure topology is provided by the spin Berry curvature.

Here we consider the Fermi energy located within the bulk states, so that in the clean regime it is simply a topologically trivial metal with a finite number of bulk propagating modes. The behavior of the normalized conductance between the two leads as a function of disorder strength is plotted in Fig. 9 (main panel) for three different Fermi energies fixed inside the bulk band structure $\left(E_{0}, E_{0} \pm \varepsilon_{1,2}\right)$ and above gap 3 . For the three cases, the conductance, high in the absence of disorder, decreases progressively upon increasing the disorder strength. Then, the three conductance curves merge and reach a plateau at $e^{2} / h$, featuring the emergence of one chiral transport channel flowing on the system's edge (bottom edge here because of the chirality in gap 3). This edge state is perfectly conducting in spite of the strong disorder, and its quantized plateau is credited to its topological robustness. Beyond a certain disorder strength, the three conductance curves drop to zero.

To complete this study, the inset of Fig. 9 compares the evolution of the conductance for different next-nearestneighbor hopping parameters, demonstrating that $t_{n}$ clearly influences the range of the disorder-induced QAH plateau by tuning the band structure topology as discussed above and renormalizing the effective electronic parameters. The inset of Fig. 8 shows that $t_{n}=0$ has a larger range of disorder-induced QAH plateaus than $t_{n}=0.3 t$. This can be attributed to the 


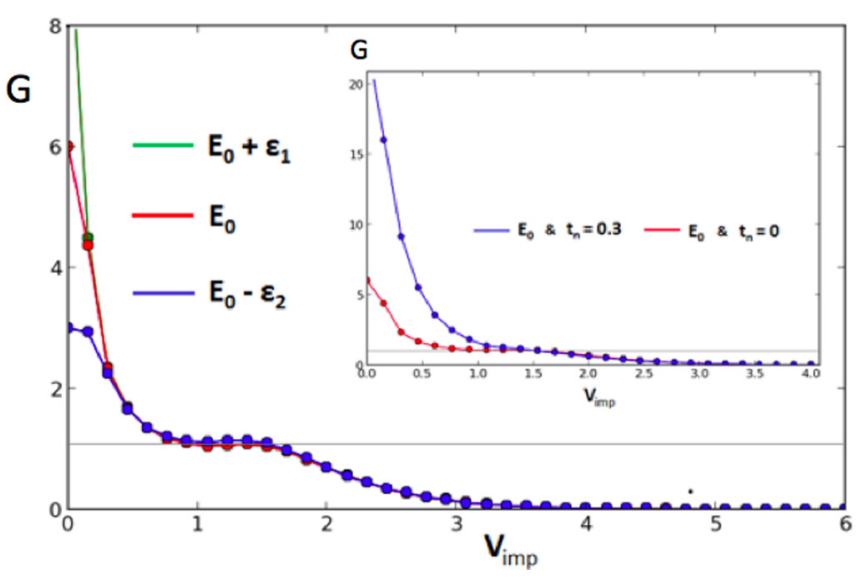

FIG. 9. (a) The conductance $G$ in units of $e^{2} / h$ as a function of the disorder strength $V_{\text {imp }}$ at fixed chemical potentials lying inside the bulk states above gap $3\left(E_{0}=2.65 t, \varepsilon_{1}=0.07 t\right.$, and $\left.\varepsilon_{2}=0.04 t\right)$; the system parameters are $W=L=20 a_{0}$ and $t_{n}=0, \Delta=t$. Inset: Comparison between conductances of two next-nearest-neighbor hopping parameters, 0 and $0.3 t$.

existence of global topological gap 3 for $t_{n}=0$ [see Fig. 6(b)], whereas it is absent for $t_{n}=0.3 t$ [this does not rule out local topological direct gap 3, as seen and discussed in the case of gap 1 in Fig. 6(c)]. For $t_{n}=0$, when the operating Fermi energy is located right above the upper limit of gap 3 in the metallic bulk band, the disorder renormalizes the topological effective mass and the carrier velocity and thus favors the emergence of the large-range disorder-induced QAH plateau when the impurity strength increases. On the other hand, there is no global topological gap 3 for $t_{n}=0.3 t$, and the range of impurity strength over which the disorder-induced plateau appears is much narrower than for $t_{n}=0$. We emphasize that while such a plateau was originally observed in the context of $\mathbb{Z}_{2}$ topological insulators $[32,33]$, it was associated with the quantum spin Hall effect. In the present case, the plateau is associated with the QAH effect, i.e., charge edge currents.

\section{CONCLUSION}

We investigated topologically protected edge transport in noncollinear, noncoplanar antiferromagnetic $3 \mathrm{Q}$ texture in the absence of spin-orbit coupling. The symmetries of the system ensure an analog to Kramers's theorem resulting in a doubly degenerate band structure. Accordingly, the noncollinear magnetic texture promotes a non-Abelian Berry curvature tensor, which is responsible for the emergence of the chiral edge states. The chirality of the topological edge modes depends on the gap in which the Fermi energy is located. We showed their robustness against disorder and geometrical defects inside the lattice and the rich phase diagram. Finally, we demonstrated that gradually tuned disorder can induce a transition in the 3Q antiferromagnet, from trivial metallic to topologically insulating regimes, through one edge mode plateau state characterized by a conductance of $e^{2} / h$.

While noncollinear coplanar antiferromagnets were recently proposed to host unconventional spin and anomalous currents [12-14], the present study highlights the potential of noncoplanar antiferromagnets for topological transport. In the former, the coexistence of SOC and noncollinear coplanar antiferromagnetic texture promotes time-reversal symmetry breaking and nonvanishing Berry curvature. In contrast, in the latter the noncoplanar antiferromagnetic texture provides both ingredients, even in the absence of SOC [28-31]. The emergence of the quantum phase of matter in frustrated quantum magnets has been the object of intense scrutiny in the past decades, with a particular focus on their magnetic phases and elementary excitations [45]. For the experimental realization of the QAH effect in antiferromagnets, magnetic pyrochlores [46] displaying an all-in, all-out spin configuration such as $\operatorname{Pr}_{2} \operatorname{Ir}_{2} \mathrm{O}_{7}$ [47] and $\mathrm{Nd}_{2} \mathrm{Ir}_{2} \mathrm{O}_{7}$ [48], possibly the Mott insulator $\mathrm{NiS}_{2}[49,50]$, and layered triangular magnets such as cobaltites [51], in particular $\mathrm{Na}_{0.5} \mathrm{CoO}_{2}$ [52], are considered as valuable candidates. All these materials exhibit a 3Q magnetic texture and insulating or bad-metal behavior and as such could support the QAH effect. We emphasize that the existence of anomalous electronic transport automatically implies the presence of the anomalous Nernst effect [53] as well as magneto-optical activity such as Kerr and Faraday effects [54]. Finally, the spin Berry curvature responsible for the electronic anomalous transport is also expected to promote magnonic anomalous transport [55], a topic we leave to future studies.

\section{ACKNOWLEDGMENTS}

The authors thank F. Piéchon, Z. Thiam, G. E. W. Bauer, K.-J. Lee, X. R. Wang, and H. Bahlouli for valuable discussions. We also acknowledge computing time on the supercomputers SHAHEEN at the KAUST Supercomputing Centre and the team assistance. This work was supported by the King Abdullah University of Science and Technology (KAUST) through the Office of Sponsored Research (OSR) under Grant No. OSR-2015-CRG4-2626. P.B.N. acknowledges the support provided by the Deanship of Scientific Research at King Fahd University of Petroleum and Minerals (KFUPM) through Project No. SR181002.
[1] J. Zelezny, H. Gao, K. Vyborny, J. Zemen, J. Masek, A. Manchon, J. Wunderlich, J. Sinova, and T. Jungwirth, Phys. Rev. Lett. 113, 157201 (2014).

[2] P. Wadley, B. Howells, J. Železný, C. Andrews, V. Hills, R. P. Campion, V. Novák, K. Olejník, F. Maccherozzi, S. S. Dhesi et al., Science 351, 587 (2016).
[3] T. Jungwirth, X. Marti, P. Wadley, and J. Wunderlich, Nat. Nanotechnol. 11, 231 (2016).

[4] V. Baltz, A. Manchon, M. Tsoi, T. Moriyama, T. Ono, and Y. Tserkovnyak, Rev. Mod. Phys. 90, 015005 (2018).

[5] L. Smejkal, Y. Mokrousov, B. Yan, and A. H. MacDonald, Nat. Phys. 14, 242 (2018). 
[6] R. S. K. Mong, A. M. Essin, and J. E. Moore, Phys. Rev. B 81, 245209 (2010).

[7] S. Ghosh and A. Manchon, Phys. Rev. B 95, 035422 (2017).

[8] P. Tang, Q. Zhou, G. Xu, and S.-C. Zhang, Nat. Phys. 12, 1100 (2016).

[9] L. Smejkal, T. Jungwirth, and J. Sinova, Phys. Status Solidi RRL 11, 1700044 (2017).

[10] S. Nakatsuji, N. Kiyohara, and T. Higo, Nature (London) 527, 212 (2015).

[11] A. K. Nayak, J. E. Fischer, Y. Sun, B. Yan, J. Karel, A. C. Komarek, C. Shekhar, N. Kumar, W. Schnelle, J. Kübler, C. Felser, and S. S. P. Parkin, Sci. Adv. 2, e1501870 (2016).

[12] H. Chen, Q. Niu, and A. H. MacDonald, Phys. Rev. Lett. 112, 017205 (2014).

[13] J. Kübler and C. Felser, Europhys. Lett. 108, 67001 (2014).

[14] J. Železný, Y. Zhang, C. Felser, and B. Yan, Phys. Rev. Lett. 119, 187204 (2017).

[15] Y. Taguchi, Y. Oohara, H. Yoshizawa, N. Nagaosa, and Y. Tokura, Science 291, 2573 (2001).

[16] K. Ohgushi, S. Murakami, and N. Nagaosa, Phys. Rev. B 62, R6065 (2000).

[17] R. Shindou and N. Nagaosa, Phys. Rev. Lett. 87, 116801 (2001).

[18] J. M. Kosterlitz, Rev. Mod. Phys. 89, 040501 (2017).

[19] F. D. M. Haldane, Rev. Mod. Phys. 89, 040502 (2017).

[20] D. Hsieh, D. Qian, L. Wray, Y. Xia, Y. Hor, R. J. Cava, and M. Z. Hasan, Nature (London) 452, 970 (2008); M. Z. Hasan and C. L. Kane, Rev. Mod. Phys. 82, 3045 (2010).

[21] C.-Z. Chang, J. Zhang, X. Feng, J. Shen, Z. C. Zhang, M. H. Guo, K. Li, Y. B. Ou, P. Wei, L.-L. Wang et al., Science 340, 167 (2013).

[22] H. Zhang, Y. Xu, J. Wang, K. Chang, and S.-C. Zhang, Phys. Rev. Lett. 112, 216803 (2014).

[23] W. Luo and X.-L. Qi, Phys. Rev. B 87, 085431 (2013); S. V. Eremeev, V. N. Menshov, V. V. Tugushev, P. M. Echenique, and E. V. Chulkov, ibid. 88, 144430 (2013).

[24] F. Katmis, V. Lauter, F. S. Nogueira, B. A. Assaf, M. E. Jamer, P. Wei, B. Satpati, J. W. Freeland, I. Eremin, D. Heiman, P. Jarillo-Herrero, and J. S. Moodera, Nature (London) 533, 513 (2016).

[25] M. P. Ghimire and M. Richter, Nano Lett. 17, 6303 (2017).

[26] F. D. M. Haldane, Phys. Rev. Lett. 61, 2015 (1988).

[27] Z. Qiao, W. Ren, H. Chen, L. Bellaiche, Z. Zhang, A. H. MacDonald, and Q. Niu, Phys. Rev. Lett. 112, 116404 (2014).

[28] J. W. F. Venderbos, M. Daghofer, J. van den Brink, and S. Kumar, Phys. Rev. Lett. 109, 166405 (2012).

[29] I. Martin and C. D. Batista, Phys. Rev. Lett. 101, 156402 (2008).

[30] H. Ishizuka and Y. Motome, Phys. Rev. B 87, 081105(R) (2013).

[31] G.-W. Chern, A. Rahmani, I. Martin, and C. D. Batista, Phys. Rev. B 90, 241102(R) (2014).
[32] J. Li, R. L. Chu, J. K. Jain, and S. Q. Shen, Phys. Rev. Lett. 102, 136806 (2009).

[33] C. W. Groth, M. Wimmer, A. R. Akhmerov, J. Tworzydlo, and C. W. J. Beenakker, Phys. Rev. Lett. 103, 196805 (2009).

[34] A. Sakuma, J. Phys. Soc. Jpn. 69, 3072 (2000).

[35] Ph. Kurz, G. Bihlmayer, K. Hirai, and S. Blugel, Phys. Rev. Lett. 86, 1106 (2001).

[36] Y. Kato, I. Martin, and C. D. Batista, Phys. Rev. Lett. 105, 266405 (2010).

[37] R. F. L. Evans, W. J. Fan, P. Chureemart, T. A. Ostler, M. O. A. Ellis, and R. W. Chantrell, J. Phys.: Condens. Matter 26, 103202 (2014).

[38] H. Yanagihara and M. B. Salamon, Phys. Rev. Lett. 89, 187201 (2002).

[39] P. B. Ndiaye, C. A. Akosa, and A. Manchon, Phys. Rev. B 95, 064426 (2017).

[40] R. Shindou and K. I. Imura, Nucl. Phys. B 720, 399 (2005).

[41] M. Gradhand, D. V. Fedorov, F. Pientka, P. Zahn, I. Mertig, and B. L. Györffy, J. Phys.: Condens. Matter 24, 213202 (2012).

[42] C. W. Groth, M. Wimmer, A. R. Akhmerov, and X. Waintal, New J. Phys. 16, 063065 (2014).

[43] J.-M. Hou and W. Chen, Sci. Rep. 5, 17571 (2015).

[44] P. W. Anderson, Phys. Rev. 109, 1492 (1958).

[45] C. D. Batista, S.-Z. Lin, S. Hayami, and Y. Kamiya, Rep. Prog. Phys. 79, 084504 (2016).

[46] J. S. Gardner, M. J. P. Gingras, and J. E. Greedan, Rev. Mod. Phys. 82, 53 (2010); S. T. Bramwell and M. J. P. Gingras, Science 294, 1495 (2001).

[47] Y. Machida, S. Nakatsuji, Y. Maeno, T. Tayama, T. Sakakibara, and S. Onoda, Phys. Rev. Lett. 98, 057203 (2007).

[48] E. Y. Ma, Y.-T. Cui, K. Ueda, S. Tang, K. Chen, N. Tamura, P. M. Wu, J. Fujioka, Y. Tokura, and Z.-X. Shen, Science 350, 538 (2015).

[49] S. Yano, D. Louca, J. Yang, U. Chatterjee, D. E. Bugaris, D. Y. Chung, L. Peng, M. Grayson, and M. G. Kanatzidis, Phys. Rev. B 93, 024409 (2016).

[50] M. Matsuura, Y. Endoh, H. Hiraka, K. Yamada, A. S. Mishchenko, N. Nagaosa, and I. V. Solovyev, Phys. Rev. B 68, 094409 (2003).

[51] N. B. Ivanova, S. G. Ovchinnikov, M. M. Korshunov, I. M. Eremin, and N. V. Kazak, Phys. Usp. 52, 789 (2009).

[52] F. L. Ning, S. M. Golin, K. Ahilan, T. Imai, G. J. Shu, and F. C. Chou, Phys. Rev. Lett. 100, 086405 (2008).

[53] M. Ikhlas, T. Tomita, T. Koretsune, M.-T. Suzuki, D. NishioHamane, R. Arita, Y. Otani, and S. Nakatsuji, Nat. Phys. 13, 1085 (2017).

[54] T. Higo, H. Man, D. B. Gopman, L. Wu, T. Koretsune, O. M. J. van't Erve, Y. P. Kabanov, D. Rees, Y. Li, M.-T. Suzuki, S. Patankar, M. Ikhlas, C. L. Chien, R. Arita, R. D. Shull, J. Orenstein, and S. Nakatsuj, Nat. Photonics 12, 73 (2018).

[55] A. Mook, J. Henk, and I. Mertig, Phys. Rev. B 90, 024412 (2014). 